\title{
The Chinese Version of the Modification of Diet in Renal Disease (MDRD) Equation is a Superior Screening Tool for Chronic Kidney Disease among Middle-aged Taiwanese than the Original MDRD and Cockcroft-Gault Equations
}

\author{
Chang-Fu Kuo ${ }^{1}$, Kuang-Hui Yu ${ }^{1}$, Yu-Ming Shen ${ }^{2}$, Lai-Chu See ${ }^{2,3}$
}

Background: Three equations have been often used to estimate glomerular filtration rate (GFR), namely, Modification of Diet in Renal Disease (MDRD), MDRD for Chinese (MDRDc), and Cockcroft-Gault (CG), for the purpose of screening individuals with chronic kidney disease (CKD). However, neither of these equations has been tested in a large Asian population. The aim of this study was to determine which equations were suitable for screening CKD in a large Taiwanese population.

Methods: The applicability of the three equations was analyzed among 32,542 participants of a health examination at Chang Gung Memorial Hospital (CGMH), Taiwan, between 2005 and 2007.

Results: Estimated glomerular filtration rate (eGFR)-MDRDc obtained the highest estimate of GFR (mean $101.5 \pm 19.2 \mathrm{ml} / \mathrm{min} / 1.73 \mathrm{~m}^{2}$ ), followed by eGFR-MDRD (mean $83.8 \pm 15.8 \mathrm{ml} / \mathrm{min} / 1.73 \mathrm{~m}^{2}$ ) and eGFR-CG (mean $79.4 \pm 29.1 \mathrm{ml} / \mathrm{min} / 1.73 \mathrm{~m}^{2}$ ). The prevalence of CKD stage 3-5 was $1.9 \%, 5.1 \%$, and $25.5 \%$ according to MDRDc, MDRD and CG equations, respectively. With respect to CKD staging, the agreement between eGFR-MDRDc and eGFR-CG (weighted kappa, $\mathrm{k}=0.22$ ) and that between eGFR-MDRD and eGFR-CG (weighted $\mathrm{k}=0.30$ ) was poor. Both the original MDRDc and MDRD indicated that subjects with risk factors for CKD had significantly lower eGFR and higher odds ratios for stage 3-5 disease than those without. Paradoxically, the mean eGFR-CG (or odds ratios) was higher (or lower) in subjects with hyperuricemia, hypertension, obesity, or metabolic syndrome than those without these risk factors.

Conclusions: The use of the CG equation in the Taiwanese population is inappropriate for screening individuals with CKD, and the MDRDc equation seems to be better for Taiwanese population.

(Biomed J 2014;37:398-405)

Key words: chronic kidney disease, glomerular filtration rate, modification of diet in renal disease equation

From the ${ }^{1}$ Division of Rheumatology, Allergy, Immunology, Chang Gung Memorial Hospital at Linkou, Chang Gung University College of Medicine, Taoyuan, Taiwan; ${ }^{2}$ Department of Public Health, College of Medicine, Chang Gung University, Taoyuan, Taiwan; ${ }^{3}$ Biostatistics Core Laboratory, Molecular Medicine Research Center, Chang Gung University, Taoyuan, Taiwan Received: Mar. 27, 2013; Accepted: Jan. 15, 2014

Correspondence to: Prof. Lai-Chu See, Department of Public Health, College of Medicine, Chang Gung University. 259, Wenhua $1^{\text {st }}$ Rd., Gueishan, Taoyuan 333, Taiwan (ROC). Tel: 886-3-2118800 ext. 5119; Fax: 886-3-2118363; E-mail: lichu@ mail.cgu.edu.tw

DOI: $10.4103 / 2319-4170.132886$ 
$\mathrm{C}$ Thronic kidney disease (CKD) is an important public health problem. CKD patients frequently have coexisting chronic medical diseases, including diabetes mellitus and hypertension, which cause complications such as renal failure and cardiovascular diseases. People in the late stage of CKD tend to have higher serum potassium $\left(\mathrm{K}^{+}\right)$level which is associated with reduced myocardial conduction velocity and accelerated repolarization. ${ }^{[1]}$ The direct and indirect costs of caring for CKD patients are enormous. ${ }^{[2]}$ Fortunately, early detection and prompt treatment of CKD may prevent or delay adverse events. ${ }^{[3]}$ However, early-stage CKD often remains undetected because of its asymptomatic nature. ${ }^{[4]}$ At-risk patients may, therefore, be unaware of their declining renal function, ${ }^{[5]}$ making early prevention difficult.

Glomerular filtration rate (GFR) is considered the best overall screening tool for assessing kidney function. Because the determination of actual GFR on the basis of insulin or radioisotope studies is expensive and impractical, the equations using plasma creatinine level have been used to estimate GFR. The National Kidney Foundation Kidney Disease Outcome Quality Initiative (K/DOQI) guidelines recommend the use of the Modification of Diet in Renal Disease (MDRD) equation ${ }^{[6]}$ or the Cockcroft-Gault (CG) equation $^{[7]}$ to estimate GFR. The CG equation was derived from a multiple linear regression analysis wherein age, weight, and serum creatinine were incorporated. Conversely, the MDRD GFR equations were correlated using log-transformed values and then reexpressed as a multiplicative linear model that contains exponents for the variables of age and serum creatinine. ${ }^{[8]}$ After being endorsed by the $\mathrm{K} / \mathrm{DOQI}$, these equations have been used in daily clinical practice. Various modifications of the MDRD equations have been proposed for different races. ${ }^{[9,10]}$ A Chinese version of the MDRD equation (MDRDc) has been developed, which differs from the MDRD equation by only a multiplier, i.e., 1.211 [estimated glomerular filtration rate $(\mathrm{eGFR})-\mathrm{MDRDc}=\mathrm{eGFR}-\mathrm{MDRD} \times 1.211] \cdot{ }^{[11]}$ However, these equations have limited applicability to populations other than Whites and Blacks, and they have not been applied to large populations.

Consistency in the use of well-validated equations for GFR prediction is of paramount importance to ensure uniformity in CKD staging, thereby facilitating the application of appropriate strategies for CKD management and the development of health policies and reimbursement schemes. ${ }^{[12]}$ In this study, we compared the results of applying the MDRDc, the MDRD, and the CG equations to estimate GFR in a large Taiwanese population.

\section{METHODS}

We analyzed the data of individuals who had undergone health examinations at Chang Gung Memorial Hos- pital (CGMH), Taiwan, between 2005 and 2007. The study protocol was approved by the Institutional Review Board of CGMH. All blood specimens obtained in this study had been analyzed at the clinical laboratory at CGMH, which is certified by the College of American Pathologists. External quality control was validated by participation in two programs: The international program run by the College of American Pathologists and the National Quality Control Program conducted by the Government of Taiwan. The internal and external quality control procedures yielded consistently satisfactory results. The levels of serum creatinine, serum albumin, serum uric acid (SUA), plasma glucose, blood urea nitrogen (BUN), and other biochemical parameters were measured using an autoanalyzer (Hitachi 7470; Hitachi, Tokyo, Japan); serum creatinine was measured by using the Jaffe method. ${ }^{[13]}$

Information regarding demographic data and disease history were collected using a structured questionnaire. Body mass index (BMI; weight in kilograms divided by the square of height in meters) was calculated for all subjects. Serum creatinine level higher than $1.4 \mathrm{mg} / \mathrm{dl}$ in men and $1.2 \mathrm{mg} / \mathrm{dl}$ in women was considered abnormal. Serum albumin level lower than $3.5 \mathrm{~g} / \mathrm{dl}$ was defined as abnormal. Hyperuricemia was defined as SUA level higher than $7.7 \mathrm{mg} / \mathrm{dl}$ in men and $6.6 \mathrm{mg} / \mathrm{dl}$ in women. Hypertension was defined as systolic blood pressure (SBP) $\geq 140 \mathrm{~mm} \mathrm{Hg}$ or diastolic blood pressure (DBP) $\geq 90 \mathrm{~mm} \mathrm{Hg}$. Hyperglycemia was defined as fasting plasma glucose level higher than $100 \mathrm{mg} / \mathrm{dl}$ or postprandial plasma glucose level higher than $140 \mathrm{mg} / \mathrm{dl}$. Metabolic syndrome was diagnosed using the modified Adult Treatment Panel (ATP) III criteria, according to the recommendations of the Health Promotion Administration of Taiwan. ${ }^{[14]}$ Hypertension defined as a component of the metabolic syndrome is $\mathrm{SBP} \geq 130 \mathrm{~mm} \mathrm{Hg}$ or DBP $\geq 85 \mathrm{~mm} \mathrm{Hg}$.

The eGFRs (eGFR-MDRDc, ${ }^{[11]}$ eGFR-MDRD, ${ }^{[6]}$ and eGFR-CG,$^{[7]}$ ) were calculated for all individuals as below.

MDRD equation corrected for Chinese subjects: ${ }^{: 11]}$

eGFR-MDRDc $=1.211 \times 170 \mathrm{sCr}^{-0.999} \times \mathrm{age}^{-0.176} \times$ $\mathrm{BUN}^{-0.170} \times \mathrm{Alb}^{+0.318} \times 0.762($ female $)$

Original MDRD equation: ${ }^{[6]}$

eGFR-MDRD $=170 \times \mathrm{sCr}^{-0.999} \times \mathrm{age}^{-0.176} \times \mathrm{BUN}^{-0.170} \times$ $\mathrm{Alb}^{+0.318} \times 0.762$ (female)

CG equation ${ }^{[7]}$ corrected for body surface area (BSA): eGFR-CG $=(140-$ age $) \times[$ body weight $(\mathrm{kg})] \times(\mathrm{BSA} / 1.73)$ $\times 0.85$ (female) $/(72 \times \mathrm{sCr})$

BSA was estimated by the Mosteller equation as follows: ${ }^{[15]}$

BSA $\left(\mathrm{m}^{2}\right)=([\text { height }(\mathrm{cm}) \times \text { weight }(\mathrm{kg})] / 3600)^{0.5}$

Moreover, CKD was classified into five stages according to the K/DOQI guidelines. ${ }^{[6]}$ When hypertension, hyperglycemia, or proteinuria was present, an eGFR of $<15 \mathrm{ml} / \mathrm{min} / 1.73 \mathrm{~m}^{2}$ was considered stage $5 \mathrm{CKD}$, 
$15-29 \mathrm{ml} / \mathrm{min} / 1.73 \mathrm{~m}^{2}$ as stage $4,30-59 \mathrm{ml} / \mathrm{min} / 1.73 \mathrm{~m}^{2}$ as stage $3,60-89 \mathrm{ml} / \mathrm{min} / 1.73 \mathrm{~m}^{2}$ as stage 2 , and an eGFR of $90 \mathrm{ml} / \mathrm{min} / 1.73 \mathrm{~m}^{2}$ as stage $1 \mathrm{CKD}$. Subjects were considered to have normal kidney function if they had an eGFR of at least $90 \mathrm{ml} / \mathrm{min} / 1.73 \mathrm{~m}^{2}$ and did not have hypertension, hyperglycemia, or proteinuria.

\section{Statistical analysis}

A scatter plot was constructed to explore the agreement between eGFR-MDRDc and eGFR-CG and between eGFR-MDRD and eGFR-CG. Weighted kappa statistics (k) were computed to compare the classification of patients according to the CKD stage between different equations. Pearson correlation coefficient $(r)$ was computed to describe the linear relationship between eGFRs and age. Independent $t$-test or Chi-square test with odds ratio (OR) was used for univariate comparison of eGFR of the three equations between subjects with and without hyperuricemia, hypertension, hyperglycemia, obesity, and metabolic syndrome, respectively. A two-sided $p$ value less than 0.05 was considered statistically significant.

\section{RESULTS}

\section{Demographics}

From 2005 through 2007, 36,223 individuals participated in the health examination program at CGMH, and 32,542 subjects $(89.8 \% ; 19,685$ men and 12,857 women) were used for the analysis because of completeness of the data. The mean ages of men and women were $51.1 \pm 12.7$ years and $53.0 \pm 12.9$ years, respectively. The mean SBP was higher in men $(129.9 \mathrm{~mm} \mathrm{Hg})$ than in women $(126.7 \mathrm{~mm} \mathrm{Hg})$; so was the mean DBP ( 80.3 vs. $75.8 \mathrm{~mm} \mathrm{Hg}$ ). Similarly, the mean serum creatinine and albumin levels were higher in men than in women. More men had hypertension (30.9\% vs. $25.7 \%$ ), hyperglycemia ( $33.1 \%$ vs. $29.4 \%$ ), and metabolic syndrome (19.6\% vs. $15.9 \%)$ than women. Only $848(4.3 \%)$ men and $426(3.4 \%)$ women had proteinuria [Table 1].

\section{Agreement in eGFR calculated using the three equations}

Because the MDRDc and MDRD equations differ only by a multiplier, i.e., 1.211, (eGFR-MDRDc $=$ eGFR-MDRD $\times 1.211$ ), scatter plots were constructed only to compare eGFR-CG with eGFR-MDRDc [Figure 1A] and eGFR-MDRD [Figure 1B]. Overall, the CKD stage defined by eGFR-CG tended to be higher than those defined by eGFR-MDRDc and eGFR-MDRD. With respect to CKD staging, the agreement was poor between eGFR-MDRDc and eGFR-CG (weighted $\mathrm{k}=0.22$ ) and between eGFR-MDRD and eGFR-CG (weighted $\mathrm{k}=0.30$ ). Concordance of CKD
Table 1: Demographic and laboratory characteristics of Taiwanese adults participating in health examinations, 2005-2007

\begin{tabular}{|c|c|c|c|}
\hline Variables & $\begin{array}{l}\text { Descriptive } \\
\text { statistics }\end{array}$ & Variables & $\begin{array}{l}\text { Descriptive } \\
\text { statistics }\end{array}$ \\
\hline \multicolumn{4}{|l|}{ Male $(n=19,685)$} \\
\hline $\begin{array}{l}\text { Age }(\text { mean } \pm \text { SD) } \\
\text { (years) }\end{array}$ & $51.1 \pm 12.7$ & BMI $\left(\mathrm{kg} / \mathrm{m}^{2}\right)$ & $24.8 \pm 3.4$ \\
\hline $20-29$ & $576(2.9 \%)$ & $\begin{array}{l}\text { Serum uric acid } \\
(\mathrm{mg} / \mathrm{dl})\end{array}$ & $6.6 \pm 1.4$ \\
\hline $30-39$ & $3164(16.1 \%)$ & Hyperuricemia (\%) & $3732(19.0)$ \\
\hline $40-49$ & $5524(28.1 \%)$ & $\begin{array}{l}\text { Plasma glucose } \\
(\mathrm{mg} / \mathrm{dl})\end{array}$ & $101.1 \pm 30.5$ \\
\hline $50-59$ & $5807(29.5 \%)$ & Hyperglycemia & $6518(33.1 \%)$ \\
\hline $60-69$ & $2800(14.2 \%)$ & $\begin{array}{l}\text { Blood pressure } \\
(\mathrm{mm} \mathrm{Hg})\end{array}$ & \\
\hline $70-79$ & $1413(7.2 \%)$ & Systolic & $129.9 \pm 17.7$ \\
\hline $80+$ & $401(2.0 \%)$ & Diastolic & $80.3 \pm 11.1$ \\
\hline Proteinuria (\%) & $848(4.3)$ & Hypertension (\%) & 6085 (30.9) \\
\hline Serum Cr (mg/dl) & $1.1 \pm 0.3$ & $\begin{array}{l}\text { Metabolic } \\
\text { syndrome (\%) }\end{array}$ & 3852 (19.6) \\
\hline Abnormal (\%) & $1157(5.9)$ & & \\
\hline Serum albumin $(\mathrm{g} / \mathrm{dl})$ & $4.6 \pm 0.3$ & & \\
\hline Abnormal (\%) & $60(0.3)$ & & \\
\hline \multicolumn{4}{|l|}{ Female $(n=12,857)$} \\
\hline $\begin{array}{l}\text { Age }(\text { mean } \pm S D) \\
\text { (years) }\end{array}$ & $53.0 \pm 12.9$ & BMI $\left(\mathrm{kg} / \mathrm{m}^{2}\right)$ & $23.8 \pm 3.7$ \\
\hline $20-29$ & $446(3.5 \%)$ & $\begin{array}{l}\text { Serum uric acid } \\
(\mathrm{mg} / \mathrm{dl})\end{array}$ & $5.1 \pm 1.3$ \\
\hline $30-39$ & $1640(12.8 \%)$ & Hyperuricemia (\%) & $1548(12.0)$ \\
\hline $40-49$ & $2906(22.6 \%)$ & Plasma glucose & $98.7 \pm 28.4$ \\
\hline $50-59$ & $4131(32.1 \%)$ & Hyperglycemia (\%) & $3783(29.4)$ \\
\hline $60-69$ & $2355(18.3 \%)$ & $\begin{array}{l}\text { Blood pressure } \\
(\mathrm{mm} \mathrm{Hg})\end{array}$ & \\
\hline $70-79$ & $1114(8.7 \%)$ & Systolic & $126.7 \pm 20.2$ \\
\hline $80+$ & $265(2.1 \%)$ & Diastolic & $75.8 \pm 10.5$ \\
\hline Proteinuria (\%) & $426(3.4)$ & Hypertension (\%) & $3308(25.7)$ \\
\hline Serum Cr (mg/dl) & $0.8 \pm 0.3$ & $\begin{array}{l}\text { Metabolic } \\
\text { syndrome }(\%)\end{array}$ & $2041(15.9 \%)$ \\
\hline Abnormal (\%) & $326(2.5 \%)$ & & \\
\hline Serum albumin $(\mathrm{g} / \mathrm{dl})$ & $4.5 \pm 0.3$ & & \\
\hline Abnormal (\%) & $40(0.3 \%)$ & & \\
\hline
\end{tabular}

Abbreviation: BMI: Body mass index

staging between eGFR-MDRDc and eGFR-CG and between eGFR-MDRD and eGFR-CG was present for $38.5 \%$ and $49.8 \%$ of the subjects, respectively.

\section{Variability of eGFR-MDRDc, eGFR-MDRD, and eGFR-CG by age}

The eGFR from the three equations decreased as age increased for both men and women. Although the Pearson correlation coefficient between eGFR-MDRDc and age $(r=-0.489)$ was slightly lower than that between eGFR-CG and age or that between eGFR-MDRD and age $(r=-0.598)$, the variability of eGFR-MDRDc by age was smaller than the variability of eGFR-CG by age [Figures $2 \mathrm{~A}, \mathrm{~B}$ ]. 


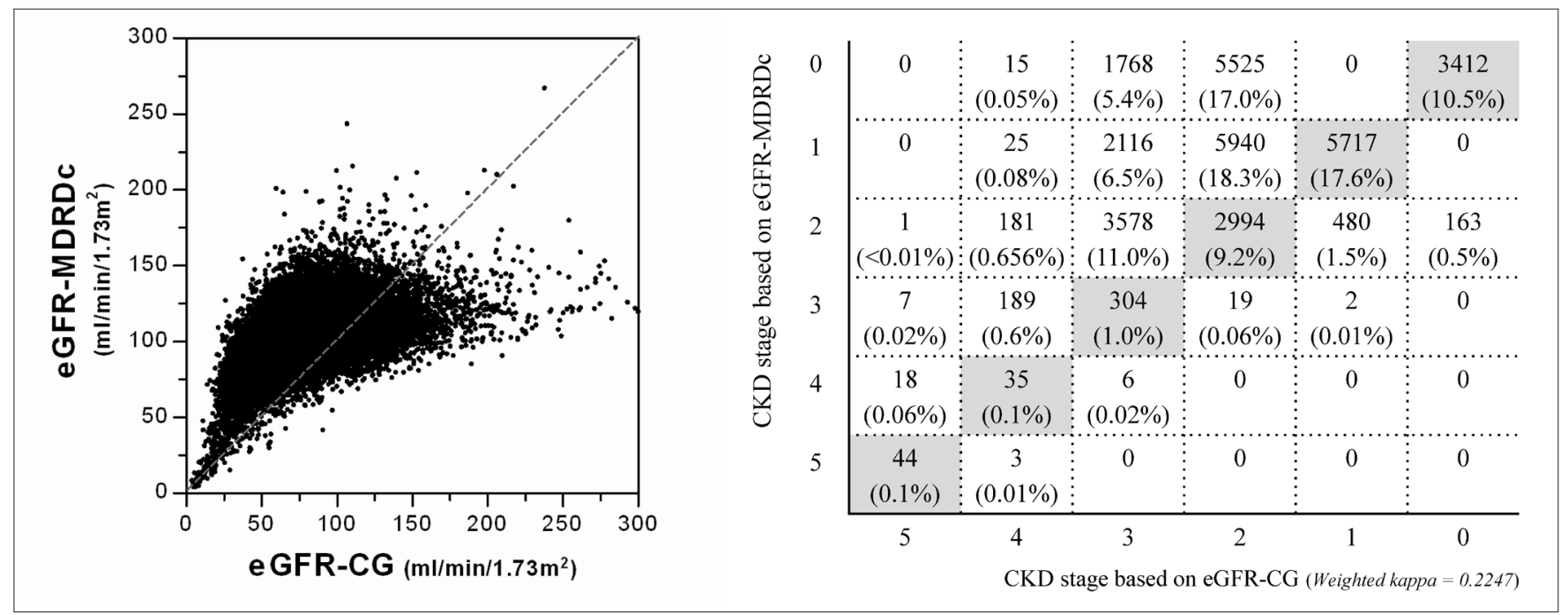

Figure 1A: Comparing eGFR-MDRDc versus eGFR-CG: eGFR and CKD stage.

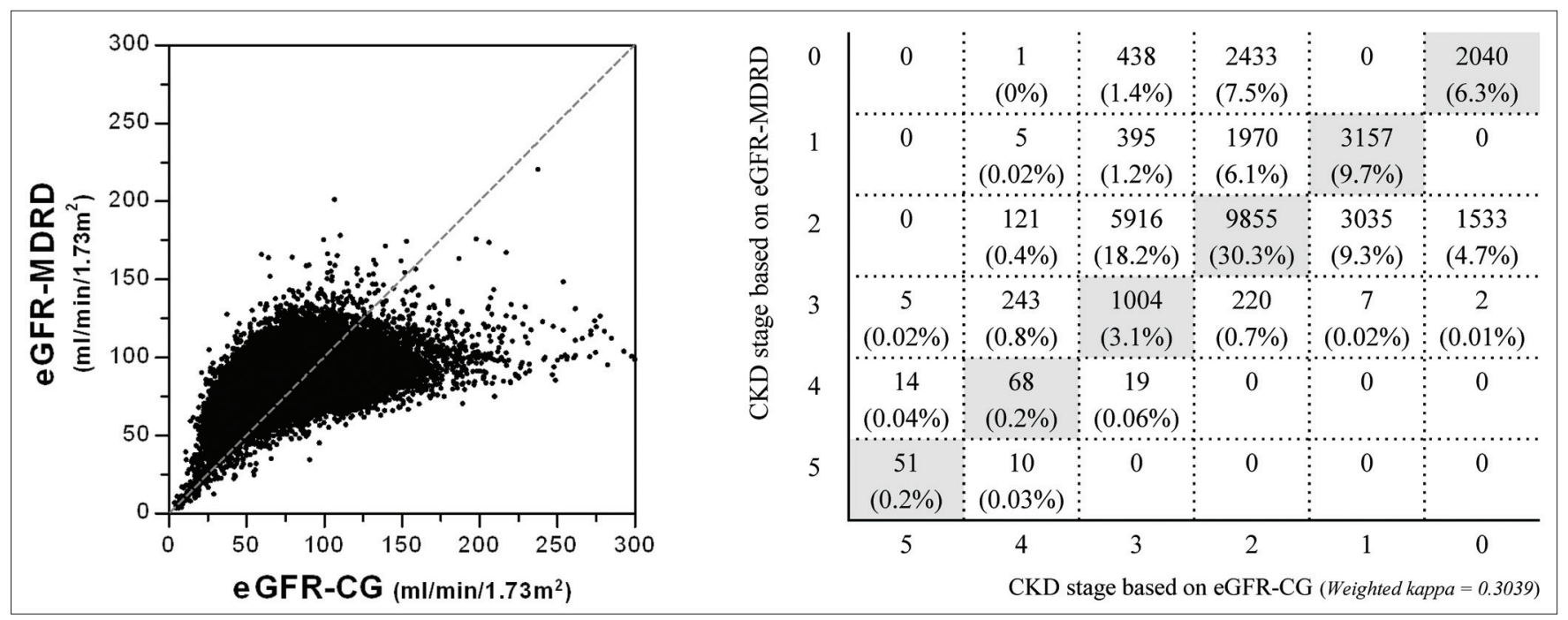

Figure 1B: Comparing eGFR-MDRD versus eGFR-CG: eGFR and CKD stage.

\section{Comparison of the equations in subjects with and without various risk factors for CKD}

Next, we compared the eGFR-MDRDc, eGFR-MDRD, and eGFR-CG determined for subjects with and without hyperuricemia, hypertension, hyperglycemia, obesity, and metabolic syndrome. In subjects with none of these conditions, the MDRDc and MDRD equations consistently generated higher values than the CG equation. The greatest difference in the GFR estimated for subjects with and without a given risk factor was observed between those with and without hyperuricemia [Table 2]. In addition, the magnitude of the difference between those with and without any of the above risk factors was higher for eGFR-MDRDc and eGFR-MDRD, as compared to eGFR-CG. Contrary to the traditional notion, eGFR-CG was significantly higher in subjects with hyperuricemia, obesity, and metabolic syndrome than in those without these conditions [Table 2].

Table 3 shows a comparison of the CKD stage determined on the basis of the three eGFR equations for patients with and without the above risk factors. The three equations differed significantly with respect to the percentage of patients identified as having stage 3-5 CKD disease (MDRDc, 1.9\%; MDRD, 5.1\%; and CG, 25.5\%). The MDRDc equation yielded the highest OR for CKD, followed by the MDRD and the CG equations, in subjects with hyperuricemia, hypertension, hyperglycemia, obesity, or metabolic syndrome [Table 3]. Contrary to the traditional view, the eGFR-CG values showed that obesity and metabolic syndrome were negatively associated with CKD (OR was 0.24 and 0.67 , respectively). 


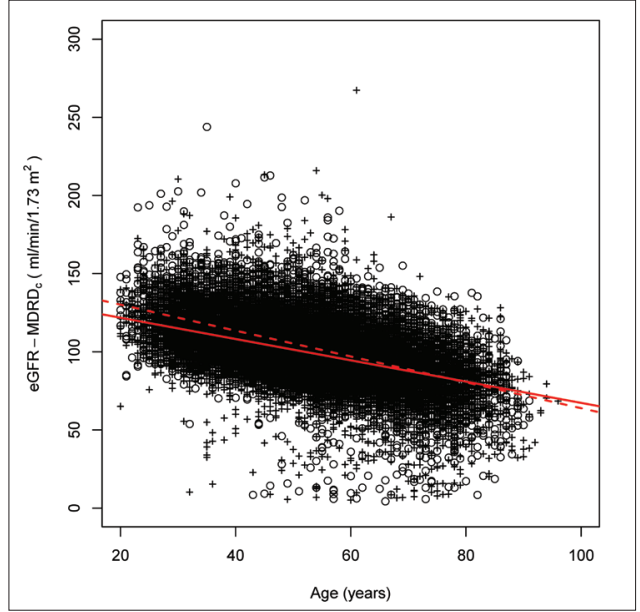

Figure 2A: Plotting eGFR-MDRDc versus age by two genders (cross and solid red line for male and open circle and broken red line for female).

\section{DISCUSSION}

This study compared the GFR estimated by three different equations in a large population of Taiwanese adults undergoing health examinations. The estimation of GFR, staging of CKD, and assignment of risk factors differed significantly among the three equations, especially in the case of the CG equation. The CG equation yielded a lower eGFR, a higher CKD stage, and a less consistent assignment of risk factors as compared to the MDRDc and MDRD equations.

Studies on Asian populations indicate that the CG equation tends to yield a higher value for the prevalence of CKD $(20.4-28.8 \%)^{[16,17]}$ than the MDRD equation $(2.5-11.9 \%)^{[16,18,19]}$ and the MDRDc equation (2.2\%). ${ }^{[20]}$ Similarly, in this study, we observed a much higher prevalence of stage 3-5 CKD when using the CG equation (25.5\%) than when using the MDRDc (1.9\%) and the MDRD equations (5.1\%). Obviously, the use of the CG equation will lead to overestimation of the CKD prevalence in the Taiwanese population.

The present study found poor agreement between the equations with respect to CKD staging. The rate of overall concordance in the classification of patients according to the CKD stage was $38.5 \%$ (kappa values $=0.22$ ) between the MDRDc and CG equations and 49.8\% (kappa values $=0.30$ ) between the MDRD and CG equations. The agreement among the eGFR equations regarding CKD staging was greater in subjects with hyperuricemia, hypertension, or metabolic syndrome than in those without these conditions.

In this study, the eGFR from the three equations showed a decline as age increased, consistent with several reviews. ${ }^{[21-23]}$ More importantly, the variability of eGFR-MDRDc by age was smaller than the variability of eGFR-CG by age, indicating that eGFR-MDRDc or eGFR-MDRD is more appropriate to screen CKD. Such high variability of eGFR-CG in young age may be due to

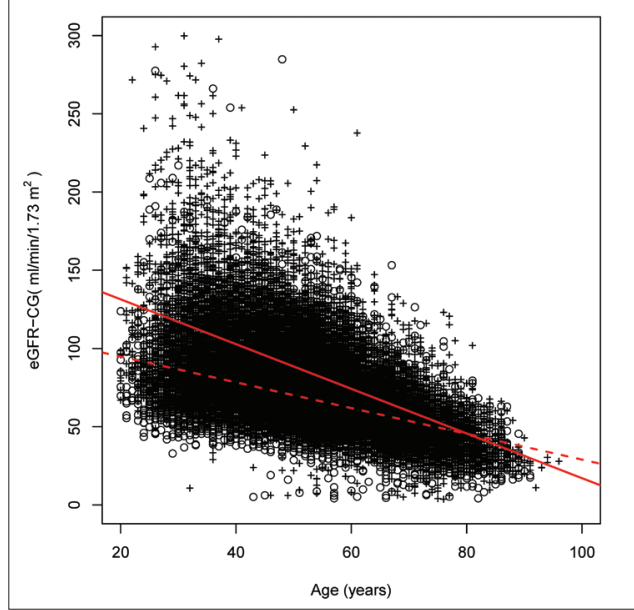

Figure 2B: Plotting eGFR-CG versus age by two genders (cross and solid red line for male and open circle and broken red line for female).

the high variance of body weight and BSA in the young. ${ }^{24]}$

Hyperuricemia $^{[25]}$ (ORs: 1.45-2.09), hyperten$\operatorname{sion}^{[26]}$ (hazard rations [HRs]: 1.0-10.4), hyperglycemia ${ }^{[27]}$ (ORs: 1.40-2.67), obesity ${ }^{[28]}$ (risk ratio [RRs]: 1.64-2.14), and metabolic syndrome ${ }^{[27]}$ (ORs: 1.16-2.32) are well-documented risk factors for CKD. In this study, both the eGFR and OR for CKD yielded by the MDRDc and MDRD equations clearly showed higher values in subjects with these risk factors than those without. However, the use of the CG equation indicated a reverse association between obesity and metabolic syndrome in terms of OR for CKD $(0.24$ and 0.67 , respectively).

Because of the much higher prevalence of CKD according to the CG equation, the low agreement between the three equations, high variability in the young, and negative risk of CKD for obesity and metabolic syndrome, we conclude that the use of the CG equation in the Taiwanese population is inappropriate and perhaps misleading for screening CKD.

The MDRDc equation, rather than the MDRD equation, should be used to estimate GFR or the CKD stage in the Taiwanese population because of the following reasons: (1) Taiwanese are similar to Chinese in terms of race, culture, diet, etc.; (2) the MDRD equation is different by a multiple of 1.210 for African-Americans ${ }^{[6]}$ and $0.808-0.881$ for Japanese ${ }^{[9,10]}(3)$ the difference in the eGFR or ORs between individuals with and without various risk factors for CKD is greater with the MDRDc equation than with the MDRD equation; and (4) the prevalence rate of CKD (1.9\%) using MDRDc equation was similar to the prevalence of clinically recognized CKD in a nationally representative cohort from 1996 to 2003 in Taiwan (1.35/100 person-years). ${ }^{[29]}$

On the other hand, there are debates about whether or not eGFR based on MDRD equation should be used to screen CKD in a large community. ${ }^{[30-33]}$ The high false-positive rate of CKD stage 3 based on MDRD equation is particularly 
Table 2: Comparison of eGFR, as determined by the different eGFR equations, in subjects with and without hyperuricemia, hypertension, hyperglycemia, obesity, and metabolic syndrome

\begin{tabular}{|c|c|c|c|}
\hline & \multicolumn{3}{|c|}{ Estimated GFR $\left(\mathrm{ml} / \mathrm{min} / 1.73 \mathrm{~m}^{2}\right)$} \\
\hline & eGFR-MDRDc & eGFR-MDRD & eGFR-CG \\
\hline Total $(n=32,542)$ & $101.5 \pm 19.2$ & $83.8 \pm 15.8$ & $79.4 \pm 29.1$ \\
\hline Male $(n=19,685)$ & $100.6 \pm 18.3$ & $83.1 \pm 15.1$ & $86.9 \pm 30.4$ \\
\hline Female $(n=12,857)$ & $102.8 \pm 20.3$ & $84.9 \pm 16.8$ & $67.7 \pm 22.4$ \\
\hline \multicolumn{4}{|l|}{ Hyperuricemia } \\
\hline No $(n=27,262)$ & $103.5 \pm 17.9$ & $85.4 \pm 14.8$ & $78.8 \pm 27.3$ \\
\hline Yes $(n=5280)$ & $91.3 \pm 22.2$ & $75.4 \pm 18.3$ & $82.5 \pm 36.8$ \\
\hline Difference & 12.1 & 10.0 & 3.7 \\
\hline$p$ value & $<0.0001$ & $<0.0001$ & $<0.0001$ \\
\hline \multicolumn{4}{|l|}{ Hypertension } \\
\hline No $(n=23,149)$ & $103.1 \pm 18.3$ & $85.1 \pm 15.1$ & $79.2 \pm 27.0$ \\
\hline Yes $(n=9393)$ & $97.5 \pm 20.6$ & $80.5 \pm 17.0$ & $79.6 \pm 33.6$ \\
\hline Difference & 5.6 & 4.6 & -0.4 \\
\hline$p$ value & $<0.0001$ & $<0.0001$ & 0.3180 \\
\hline \multicolumn{4}{|l|}{ Hyperglycemia } \\
\hline No $(n=22,241)$ & $103.3 \pm 18.1$ & $85.3 \pm 15.0$ & $80.0 \pm 27.8$ \\
\hline Yes $(n=10,301)$ & $97.6 \pm 20.7$ & $80.6 \pm 17.1$ & $78.1 \pm 31.6$ \\
\hline Difference & 5.7 & 4.7 & 1.9 \\
\hline$p$ value & $<0.0001$ & $<0.0001$ & $<0.0001$ \\
\hline \multicolumn{4}{|l|}{ Obesity } \\
\hline No $(n=25,747)$ & $102.3 \pm 19.0$ & $84.5 \pm 15.7$ & $73.3 \pm 23.3$ \\
\hline Yes $(n=6787)$ & $98.5 \pm 19.4$ & $81.3 \pm 16.1$ & $102.3 \pm 36.5$ \\
\hline Difference & 3.8 & 3.1 & -29.0 \\
\hline$p$ value & $<0.0001$ & $<0.0001$ & $<0.0001$ \\
\hline \multicolumn{4}{|l|}{ Metabolic syndrome } \\
\hline No $(n=26,531)$ & $102.6 \pm 18.4$ & $84.7 \pm 15.2$ & $76.9 \pm 26.1$ \\
\hline Yes $(n=6011)$ & $96.5 \pm 21.5$ & $79.7 \pm 17.7$ & $90.2 \pm 37.8$ \\
\hline Difference & 6.2 & 5.1 & -13.4 \\
\hline$p$ value & $<0.0001$ & $<0.0001$ & $<0.0001$ \\
\hline
\end{tabular}

Abbreviations: GFR: glomerular filtration rate; MDRD: modification of diet in renal disease. Hyperuricemia defined as serum uric acid $>7.7 \mathrm{mg} / \mathrm{dl}$ in men or $>6.6 \mathrm{mg} / \mathrm{dl}$ in women; hypertension defined as systolic blood pressure $\geq 140 \mathrm{~mm} \mathrm{Hg}$ or diastolic blood pressure $\geq 90 \mathrm{~mm} \mathrm{Hg}$; hyperglycemia defined as fasting plasma glucose $\geq 100 \mathrm{mg} / \mathrm{dl}$ or postprandial plasma glucose $\geq 140 \mathrm{mg} / \mathrm{dl}$; metabolic syndrome defined by the criteria proposed by the Health Promotion Administration, Taiwan, 2003 ${ }^{[14]}$

worrisome because it leads to unnecessary investigations, referrals, cost, and anxiety. ${ }^{[30]}$ Due to this, many recommendations have been made to screen CKD using eGFR with MDRD equation in order alert individuals who are at risk of developing renal problem or cardiovascular diseases. Abnormal albuminuria $\mathrm{a}^{[33]}$ and proteinuria ${ }^{[31]}$ should be added as the requirement for CKD stage 3. Individuals with CKD stage 4 and 5 based on eGFR should be referred. ${ }^{[34]}$ Early intervention strategies, such as diet control, can then be implemented to prevent the development of adverse renal problem.

Unfortunately, Taiwan has the highest incidence of end-stage renal disease (ESRD) in the world and a relatively high prevalence and incidence of CKD. ${ }^{[29]}$ Since 1996, a free
Table 3: Comparison of CKD status, as determined by the different eGFR equations, in subjects with and without hyperuricemia, hypertension, hyperglycemia, obesity, and metabolic syndrome

\begin{tabular}{|c|c|c|c|}
\hline & \multicolumn{3}{|c|}{ CKD stage $3-5$} \\
\hline & eGFR-MDRDc & eGFR-MDRD & eGFR-CG \\
\hline Total $(n=32,542)$ & $627(1.9 \%)$ & $1643(5.1 \%)$ & $8290(25.5 \%)$ \\
\hline Male $(n=19,685)$ & $356(1.8 \%)$ & $983(5.0 \%)$ & $3278(16.7 \%)$ \\
\hline Female $(n=12,857)$ & $271(2.1 \%)$ & $660(5.1 \%)$ & $5012(39.0 \%)$ \\
\hline \multicolumn{4}{|l|}{ Hyperuricemia } \\
\hline No $(n=27,262)$ & $254(0.9 \%)$ & $833(3.1 \%)$ & $6824(25.0 \%)$ \\
\hline Yes $(n=5280)$ & $373(7.1 \%)$ & $810(15.3 \%)$ & $1466(27.8 \%)$ \\
\hline Odds ratio & 8.08 & 5.75 & 1.15 \\
\hline$p$ value & $<0.0001$ & $<0.0001$ & $<0.0001$ \\
\hline Adjusted OR* & 7.12 & 6.31 & 1.04 \\
\hline$p$ value & $<0.0001$ & $<0.0001$ & 0.3765 \\
\hline \multicolumn{4}{|l|}{ Hypertension } \\
\hline No $(n=23,149)$ & $286(1.2 \%)$ & $836(3.6 \%)$ & $5552(24.0 \%)$ \\
\hline Yes $(n=9393)$ & $341(3.6 \%)$ & $807(8.6 \%)$ & $2738(29.2 \%)$ \\
\hline Odds ratio & 3.01 & 2.51 & 1.30 \\
\hline$p$ value & $<0.0001$ & $<0.0001$ & $<0.0001$ \\
\hline Adjusted OR* & 1.61 & 1.43 & 0.68 \\
\hline$p$ value & $<0.0001$ & $<0.0001$ & $<0.0001$ \\
\hline \multicolumn{4}{|l|}{ Hyperglycemia } \\
\hline No $(n=22,241)$ & $250(1.1 \%)$ & $745(3.4 \%)$ & $5257(23.6 \%)$ \\
\hline Yes $(n=10,301)$ & $377(3.7 \%)$ & $898(8.7 \%)$ & $3033(29.4 \%)$ \\
\hline Odds ratio & 3.34 & 2.76 & 1.35 \\
\hline$p$ value & $<0.0001$ & $<0.0001$ & $<0.0001$ \\
\hline Adjusted OR* & 1.72 & 1.45 & 0.58 \\
\hline$p$ value & $<0.0001$ & $<0.0001$ & $<0.0001$ \\
\hline \multicolumn{4}{|l|}{ Obesity } \\
\hline No $(n=25,747)$ & $453(1.8 \%)$ & $1159(4.5 \%)$ & $7665(29.8 \%)$ \\
\hline Yes $(n=6787)$ & $174(2.6 \%)$ & $484(7.1 \%)$ & $624(9.2 \%)$ \\
\hline Odds ratio & 1.47 & 1.63 & 0.24 \\
\hline$p$ value & $<0.0001$ & $<0.0001$ & $<0.0001$ \\
\hline Adjusted OR* & 1.40 & 1.67 & 0.11 \\
\hline$p$ value & 0.0007 & $<0.0001$ & $<0.0001$ \\
\hline \multicolumn{4}{|l|}{ Metabolic syndrome } \\
\hline No $(n=26,531)$ & $364(1.4 \%)$ & $1056(4.0 \%)$ & $7130(26.8 \%)$ \\
\hline Yes $(n=6011)$ & $263(4.5 \%)$ & $587(10.0 \%)$ & $1160(19.7 \%)$ \\
\hline Odds ratio & 3.37 & 2.68 & 0.67 \\
\hline$p$ value & $<0.0001$ & $<0.0001$ & $<0.0001$ \\
\hline Adjusted OR* & 2.58 & 2.15 & 0.33 \\
\hline$p$ value & $<0.0001$ & $<0.0001$ & $<0.0001$ \\
\hline
\end{tabular}

Abbreviations: GFR: glomerular filtration rate; MDRD: modification of diet in renal disease; OR: odds ratio; CKD: chronic kidney disease. Hyperuricemia defined by serum uric acid $>7.7 \mathrm{mg} / \mathrm{dl}$ in men or $>6.6$ $\mathrm{mg} / \mathrm{dl}$ in women; hypertension defined by systolic blood pressure $140 \mathrm{~mm} \mathrm{Hg}$ or diastolic blood pressure $90 \mathrm{~mm} \mathrm{Hg}$; hyperglycemia defined by fasting plasma glucose $100 \mathrm{mg} / \mathrm{dl}$ or postprandial plasma glucose $140 \mathrm{mg} / \mathrm{dl}$; metabolic syndrome defined by the criteria proposed by the Health Promotion Administration, Taiwan, 2003; $;^{[14]}$ *adjusted for age and sex

health examination annually for individuals aged $>65$ years and every 3 years for individuals aged $>40$ years has been sponsored by Taiwan National Health Insurance program. ${ }^{[35]}$ 
Hence, we recommend the Chinese equation rather than the MDRD equation to screen CKD for the Taiwanese population.

There are several limitations to this study. First, the eGFR calculated using plasma creatinine may not reflect the actual GFR, especially in early stages of CKD. Fitting a new equation for measurement of the GFR on the basis of insulin or radioisotope studies is necessary to verify the appropriateness of the MDRDc equation for the Taiwanese population. Second, the possibility of a significant misclassification of CKD on the basis of eGFR cannot be excluded. In particular, CKD stage 2 (eGFR $60-89 \mathrm{ml} / \mathrm{min} / 1.73 \mathrm{~m}^{2}$ ) requires evidence of structural damage, as per the standard definition of $\mathrm{CKD},{ }^{[6]}$ but information about structural damage was not available in the health examination data. Third, our data are cross-sectional in nature.

The strengths of the current study include the analysis of data from a large sample of community-dwelling adults, which ensures the generalizability of the findings, and its approach of comparing the results from the most widely used tools for the clinical assessment of the renal function. To our knowledge, this study is the first to apply the MDRDc equation and compare it to the MDRD and CG equations. Our findings also indicate that the use of the CG equation for CKD staging is misleading in the Taiwanese population and may adversely affect resource allocation in health care systems. This study has shown that the choice of eGFR equation can greatly affect the results of CKD epidemiology and that the MDRDc equation is more suitable for the Taiwanese population.

\section{Conclusion}

The use of the CG equation to estimate GFR in the Taiwanese population is inappropriate and perhaps misleading for screening CKD because of the high prevalence of CKD based on the CG equation, low agreement of the three equations, and negative risk of obesity or metabolic syndrome on CKD. The Chinese version of eGFR-MDRD equation (eGFR-MDRDc) is therefore recommended for the Taiwanese population. Further studies which measure actual GFR on the basis of insulin or radioisotope studies are necessary to confirm these results.

\section{Acknowledgments}

The authors would like to thank the Ministry of Education, Taiwan (EMRPD170101) and Chang Gung Memorial Hospital (CMRPG370401) for financially supporting this research.

\section{REFERENCES}

1. Hsieh MF, Wu IW, Lee CC, Wang SY, Wu MS. Higher serum potassium level associated with late stage chronic kidney disease.
Chang Gung Med J 2011;34:418-25.

2. Khan S, Amedia CA Jr. Economic burden of chronic kidney disease. J Eval Clin Pract 2008;14:422-34.

3. Locatelli F, Vecchio LD, Pozzoni P. The importance of early detection of chronic kidney disease. Nephrol Dial Transplant 2002;17 Suppl 11:2-7.

4. Ruggenenti P, Schieppati A, Remuzzi G. Progression, remission, regression of chronic renal diseases. Lancet 2001;357:1601-8.

5. Minutolo R, De Nicola L, Mazzaglia G, Postorino M, Cricelli C, Mantovani LG, et al. Detection and awareness of moderate to advanced CKD by primary care practitioners: A cross-sectional study from Italy. Am J Kidney Dis 2008;52:444-53.

6. Levey AS, Coresh J, Balk E, Kausz AT, Levin A, Steffes MW, et al. National Kidney Foundation practice guidelines for chronic kidney disease: Evaluation, classification, and stratification. Ann Intern Med 2003;139:137-47.

7. Cockcroft DW, Gault MH. Prediction of creatinine clearance from serum creatinine. Nephron 1976;16:31-41.

8. Spruill WJ, Wade WE, Cobb HH. Comparison of estimated glomerular filtration rate with estimated creatinine clearance in the dosing of drugs requiring adjustments in elderly patients with declining renal function. Am J Geriatr Pharmacother 2008;6:153-60.

9. Imai E, Horio M, Nitta K, Yamagata K, Iseki K, Tsukamoto Y, et al. Modification of the Modification of Diet in Renal Disease (MDRD) Study equation for Japan. Am J Kidney Dis 2007;50:927-37.

10. Matsuo S, Imai E, Horio M, Yasuda Y, Tomita K, Nitta K, et al. Revised equations for estimated GFR from serum creatinine in Japan. Am J Kidney Dis 2009;53:982-92.

11. Ma YC, Zuo L, Chen JH, Luo Q, Yu XQ, Li Y, et al. Modified glomerular filtration rate estimating equation for Chinese patients with chronic kidney disease. J Am Soc Nephrol 2006;17:2937-44.

12. Zhang QL, Rothenbacher D. Prevalence of chronic kidney disease in population-based studies: Systematic review. BMC Public Health 2008;8:117-30.

13. Spencer K. Analytical reviews in clinical biochemistry: The estimation of creatinine. Ann Clin Biochem 1986;23:1-25.

14. Tan CE, Ma S, Wai D, Chew SK, Tai ES. Can we apply the national cholesterol education program adult treatment panel definition of the metabolic syndrome to Asians? Diabetes Care 2004;27:1182-6.

15. Mosteller RD. Simplified calculation of body-surface area. N Engl J Med 1987;317:1098.

16. Chen J, Wildman RP, Gu D, Kusek JW, Spruill M, Reynolds K, et al. Prevalence of decreased kidney function in Chinese adults aged 35 to 74 years. Kidney Int 2005;68:2837-45.

17. Konta T, Hao Z, Abiko H, Ishikawa M, Takahashi T, Ikeda A, et al. Prevalence and risk factor analysis of microalbuminuria in Japanese general population: The Takahata study. Kidney Int 2006;70:751-6.

18. Li ZY, Xu GB, Xia TA, Wang HY. Prevalence of chronic kidney disease in a middle and old-aged population of Beijing. Clin Chim Acta 2006;366:209-15.

19. Shankar A, Klein R, Klein BE. The association among smoking, heavy drinking, and chronic kidney disease. Am J Epidemiol 2006;164:263-71. 
20. See LC, Kuo CF, Chuang FH, Shen YM, Ko YS, Chen YM, et al. Hyperuricemia and metabolic syndrome: Associations with chronic kidney disease. Clin Rheumatol 2011;30:323-30.

21. Silva FG. The aging kidney: A review-Part I. Int Urol Nephrol 2005;37:185-205.

22. Epstein M. Aging and the kidney. J Am Soc Nephrol 1996;7:1106-22.

23. Martin JE, Sheaff MT. Renal ageing. J Pathol 2007;211:198-205.

24. Michels WM, Grootendorst DC, Verduijn M, Elliott EG, Dekker FW, Krediet RT. Performance of the Cockcroft-Gault, MDRD, and new CKD-EPI formulas in relation to GFR, age, and body size. Clin J Am Soc Nephrol 2010;5:1003-9.

25. Obermayr RP, Temml C, Gutjahr G, Knechtelsdorfer M, Oberbauer R, Klauser-Braun R. Elevated uric acid increases the risk for kidney disease. J Am Soc Nephrol 2008;19:2407-13.

26. Haroun MK, Jaar BG, Hoffman SC, Comstock GW, Klag MJ, Coresh J. Risk factors for chronic kidney disease: A prospective study of 23,534 men and women in Washington County, Maryland. J Am Soc Nephrol 2003;14:2934-41.

27. Chen J, Gu D, Chen CS, Wu X, Hamm LL, Muntner P, et al. Association between the metabolic syndrome and chronic kidney disease in Chinese adults. Nephrol Dial Transplant 2007;22:1100-6.

28. Chertow GM, Hsu CY, Johansen KL. The enlarging body of evidence: Obesity and chronic kidney disease. J Am Soc Nephrol 2006;17:1501-2.

29. Kuo HW, Tsai SS, Tiao MM, Yang CY. Epidemiological features of CKD in Taiwan. Am J Kidney Dis 2007;49:46-55.

30. Glassock RJ, Winearls C. Screening for CKD with eGFR: Doubts and dangers. Clin J Am Soc Nephrol 2008;3:1563-8.

31. Cheung CK, Bhandari S. Perspectives on eGFR reporting from the interface between primary and secondary care. Clin J Am Soc Nephrol 2009;4:258-60.

32. Glassock RJ, Winearls C. An epidemic of chronic kidney disease: Fact or fiction? Nephrol Dial Transplant 2008;23:1117-21.

33. De Jong PE, Gansevoort RT. Fact or fiction of the epidemic of chronic kidney disease-let us not squabble about estimated GFR only, but also focus on albuminuria. Nephrol Dial Transplant 2008;23:1092-5.

34. Richards N, Harris K, Whitfield M, O'Donoghue D, Lewis R, Mansell M, et al. Primary care-based disease management of chronic kidney disease (CKD), based on estimated glomerular filtration rate (eGFR) reporting, improves patient outcomes. Nephrol Dial Transplant 2008;23:549-55.

35. National Health Insurance Administratoin, National Health Insurance Profile, Taiwan. 